\title{
Expression of claudin-1 and its relationship with lymphatic microvessel generation in hypopharyngeal squamous cell carcinoma
}

\author{
W.J. Li ${ }^{1,2}$, Z.L. Zhang' ${ }^{2}$, X.M. Yu ${ }^{1}$, X.L. Cai ${ }^{1}$, X.L. Pan $^{1}$ and X.Y. Yang ${ }^{3}$ \\ ${ }^{1}$ Qilu Hospital of Shandong University, Jinan, Shandong Province, China \\ 2Department of Otolaryngology, Tai'an City Central Hospital, Tai'an, \\ Shandong Province, China \\ 'Department of Central Laboratory, Tai'an City Central Hospital, Tai'an, \\ Shandong Province, China \\ Corresponding author: X.L. Pan \\ E-mail: ta3709@126.com
}

Genet. Mol. Res. 14 (4): 11814-11826 (2015)

Received February 27, 2015

Accepted June 26, 2015

Published October 2, 2015

DOI http://dx.doi.org/10.4238/2015.October.2.15

ABSTRACT. We investigated the relationship between claudin-1 and micro-lymphatic vessel density (MLVD) by detecting claudin-1 and protein D2-40 expression in cancer tissue specimens obtained from 97 patients with hypopharyngeal squamous cell carcinoma (HSCC). We also explored the correlation between the expression of these proteins and clinical tumor stage, pathological grading, and clinical prognosis in the patients. Moreover, we studied the mechanism of lymph node metastasis in HSCC, thereby providing information for treating HSCC and inhibiting lymph node metastasis. We detected levels of claudin-1 and protein D240 expression in cancer tissue from 97 patients with HSCC and paratumor tissue from 90 patients by immunohistochemistry; we analyzed the correlation between markers and clinicopathological features by using the Pearson chi-square test and conducted survival analysis by the log-rank test. Claudin-1 expression was high in HSCC and was related to tumor differentiation and lymph node metastasis; Kaplan-Meier analysis showed 
that claudin-1 expression was related to patient survival rate $(P=0.012)$. There was a significant relationship between MLVD in the tissues adjacent to the carcinoma and the indices of histopathological grade, clinical stage, and lymph node metastasis. There was also a positive correlation between claudin-1 expression and MLVD. High expression of claudin-1 might induce the generation of tumor lymphatic vessels, which increases metastasis in the lymph node. Because claudin- 1 is related to patient survival rate, it may be useful as a monitoring index for postoperative HSCC and might be a new target for treating the disease.

Key words: Claudin-1; D2-40; Hypopharyngeal squamous cell carcinoma

\section{INTRODUCTION}

The mechanism by which hypopharyngeal squamous cell carcinoma (HSCC) exerts its adverse biological characteristics is obscure and difficult to elucidate. The hypopharynx contains a rich network of lymphatic vessels, which facilitates cervical lymph node metastasis. Johnson et al. (1994) discovered that in patients with HSCC who were not receiving treatment, the rate of cervical lymph node metastasis in the clinical stage $\mathrm{N}_{1}-\mathrm{N}_{2}$ was $65-80 \%$. However, postoperative pathological diagnosis of patients in the clinical stage $\mathrm{N}_{0}$ who had undergone neck node dissection showed a cervical lymph node metastasis rate of 30-40\% (Mazzone et al., 1990). Cervical lymph node metastasis has become one of the main causes of treatment failure in HSCC (Pingree et al., 1987), and is the most important factor influencing the prognosis of patients with HSCC; the prognosis for HSCC has become one of the worst in head and neck cancer. Through studying the HSCC cervical lymph node metastasis mechanism, we discovered the factors that influence the generation of HSCC lymph metastases. If we could evaluate the potential for metastasis in HSCC patients following recovery, we could design special programs for more effective clinical treatment.

Tight junctions (TJs), which function as barriers and palisades, exist in body epithelial and endothelial cells and maintain the hemogenesis and polarity of cells. As an important membrane junction complex in cells, the TJ is composed of three membrane proteins: occludin, claudin, and the junctional adhesion molecules. Changes in the expression level and structure of claudin, which is the main membrane protein in TJs (Heiskala et al., 2001), directly influence the structure and function of TJs at the membrane surface. Claudin is expressed abnormally in a variety of malignant tumors; it changes the normal structure and function of TJs, causing cells to lose polarity, decreasing their adhesion capacity and promoting the invasiveness and metastasis potential of tumor cells (Harhaj and Antonetti, 2004).

Claudin-1 is expressed differently in different tumors (upregulation or downregulation). Studies have shown that claudin-1 is overexpressed in renal cell carcinoma (Fritzsche et al., 2008), ovarian cancer (Soini and Talvensaari-Mattila, 2006), and colorectal cancer (Huo et al., 2009), promoting the generation and development of tumors. However, in studies on breast cancer (Tökés et al., 2005) and lung adenocarcinoma (Chao et al., 2009), claudin-1 expression decreased. It has been proved that there is a significant correlation between the differential expression of claudin-1 and tumor generation and development.

Tumor metastasis is a complex process that is influenced by many factors and comprises three steps: adhesion, degradation, and migration. The most common tumor metastatic pathways are vascular and lymphatic. It has been confirmed that vascular and lymphatic generation are 
very important in tumor growth, invasion, and metastasis. Since Folkman (1995) confirmed that nutrition for tumor growth and invasion was provided by new microvessels, studies on tumor vessel metastasis have made substantial advances; lymphatic vessel growth also plays an important role in tumor metastasis. With the discovery of the specific antibody to lymphatic endothelial cells, lymphatic microvessels as the channel for invasive tumor metastasis have received increasing attention. Micro-lymphatic vessel density (MLVD) has become the most important factor for accessing malignant tumor metastasis (Kumada et al., 2004).

Recent studies have found that D2-40 is the most specific marker of lymphatic vessels. As the monoclonal antibody that specifically binds to carcinoembryonic antigen M2A, D2-40 can recognize the sialoglycoprotein expressed in lymphatic endothelial cells (Kahn et al., 2002); it only develops color in tiny lymphatic channels with monolayer endothelial cells but never in big lymphatic vessels. Not only can it help in the recognition of small lymphatic vessels, it also specifically distinguishes between lymphogenic and angiogenic cerebral tumors (Liang et al., 2005). Therefore, D2-40 is used widely for studying the generation of tumor lymphatic vessels.

By detecting the expression levels of tumor tissue claudin-1 and D2-40 protein in 97 patients with HSCC, we investigated the relationship between claudin-1 and MLVD with respect to HSCC invasion, metastasis, and prognosis. We explored the mechanism of metastasis in HSCC and provided evidence for inhibiting lymph node metastasis as a means of treating HSCC.

\section{MATERIAL AND METHODS}

\section{Cases and specimens}

We collected HSCC specimens from patients undergoing surgical treatment in the Otorhinolaryngology Department of Qilu Hospital at Shandong University from January 2008 to April 2011. We took adjacent tissues from 90 cases and mucosa with normal morphology at a distance of at least $2 \mathrm{~cm}$ from the surgical margin. All the specimens were fixed in formalin and paraffin-embedded according to conventional methods. None of patients received radiotherapy, chemotherapy, etc., before surgical treatment. All the patients underwent a standard operation and the surgical margins were negative. After the operation, patients received radiotherapy at dosage of 50-65 Gy. All the cases were pathologically staged and classified according to the TNM staging criteria of UICC, 2002. The patient follow-up time was 3-5 years and all the cases were grouped according to the following criteria: age, gender, tobacco consumption (little or none, or excess), alcohol consumption (little or none, or too much), differentiation, TNM stage, and lymph node metastases. All the specimen collection procedures were audited by the institutional review board of Qilu Hospital at Shandong University before collection, and patients or their family members were informed.

\section{Immunohistochemistry}

Claudin-1 (ZA-0365) and D2-40 (ZM-0465) were obtained from the Beijing ZSGB-BIO Company, Beijing, China. We conducted staining according to the following steps: 1) tissue sections were baked at $62^{\circ} \mathrm{C}$ for $30 \mathrm{~min}$ in an oven. 2) The sections were dewaxed in xylene and hydrated by placing successively in $100,95,70,50$, and $30 \%$ ethanol solutions, then washed with $0.01 \mathrm{M}$ phosphate-buffered saline (PBS); the entire procedure was repeated three times. 3) We carried out thermal remediation on the tissue antigens with ethylenediaminetetraacetic acid repair liquid $(\mathrm{pH}$ 
8.0) in a microwave oven for $15 \mathrm{~min}$, then cut the power and allowed natural cooling to a temperature lower than $30^{\circ} \mathrm{C}$, and washed the samples three times with PBS. 4) We added $3 \% \mathrm{H}_{2} \mathrm{O}_{2}$ in drops, incubated at $25^{\circ} \mathrm{C}$ for $10 \mathrm{~min}$, and washed in PBS three times. 5) We added the primary antibody (specific to claudin-1 and D2-40) overnight at $4^{\circ} \mathrm{C}$, washed with PBS for 5 min and repeated the total procedure three times. 6) We added agent 1 (polymer adjuvant), incubated at $37^{\circ} \mathrm{C}$ for $20 \mathrm{~min}$, and washed with PBS three times. 7) We then added agent 2 (goat anti-mouse IgG polymer labeled with horseradish peroxidase) and incubated at $37^{\circ} \mathrm{C}$ for $30 \mathrm{~min}$, then washed with PBS three times. 8) The samples were reacted with 3,3'-diaminobenzidine chromogenic reagent and counterstained with hematoxylin. PBS was used as a negative control instead of the primary antibody.

\section{Analysis of results}

Two doctors with extensive experience in pathology observed the staining results independently. Claudin-1-positive particles were yellow or brown and were located in the membrane or cytoplasm. Referring to the standards modified by Kojima (Ishida et al., 2009), ten randomly selected areas of each tissue slide were analyzed using high-power field (400X) in counting 100 cells per field. We conducted semi-quantitative assays according to comprehensive staining intensity and positive cells. The marking standards and staining intensities are shown in Table 1. Immunoreaction was evaluated by the percentage of cells staining positively. The values were given for semiquantitative evaluation: 0 (0-5\% positivity), 1 (6-20\% positivity), 2 (21-40\% positivity), 3 (41-60\% positivity), 4 (61-80\% positivity), and 5 (81-100\% positivity). With the staining intensity and the scores, those with total scores from 0 to 2 were considered negative (-), those with a total scores of 2 were weakly positive $(+)$, those with scores from 3 to 4 were positive $(++)$, and those with scores 5 were strongly positive $(+++)$. All the results were obtained by double-blinded film reading. Those with scores greater than 3 exhibited high expression (Figure 1A); those with scores of 2 or less exhibited low expression (Figure 1B).

Table 1. Staining intensities and percentages of positive cells.

\begin{tabular}{lc}
\hline Marking standard & Scores \\
\hline Staining intensity & 0 \\
Negative & 1 \\
Weakly positive & 2 \\
Positive & 3 \\
$\quad$ Strongly positive & 0 \\
Number of positive cells & 1 \\
$<10 \%$ & 2 \\
$10-40 \%$ & 3 \\
$40-70 \%$ & 3 \\
$>70 \%$ & \\
\hline
\end{tabular}

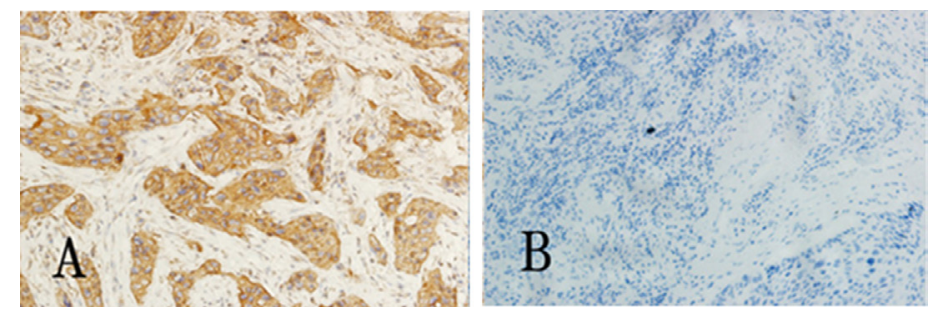

Figure 1. A. positive expression of claudin-1 (200X). B. Negative expression (200X). 


\section{MLVD counting: D2-40-stained lymphatic microvessel endothelial cells}

Positive expression in the cytoplasm is shown as brown particles. Most of the walls of the lymphatic microvessels were positively stained and comprised a monolayer of endothelial cells, which was thin and lacked a muscular layer or any erythrocytes, and the shapes were irregular, with round, expanding, or blocked, stripy appearance. Parts of the lymphatic microvessel walls were incomplete. Referring to the methods reported by MAEDA etc. (Wang et al., 1999), we first observed the slides under a low-power lens (100X) and confirmed the "hot spot" with the highest density of lymphatic microvessels in the tumor tissue. Second, we counted the number of lymphatic microvessels in each section under a high-power lens (400X) by taking single or agminated lymphatic endothelial cells or clusters without a lumen, which were stained yellow or brown, as independent lymphatic vessels, regardless of connections. The counting was undertaken by two doctors from the department of pathology, who were blinded to the clinical pathological data of the patients (the double-blind method). We then took the means of the two counts. As a result, we took five average values of the lymphatic microvessel numbers under the high-power lens (400X) as the MLVD.

\section{Statistical analysis}

Data were analyzed statistically using the SPSS 17.0 software. Data from two groups were compared with an independent sample Mann-Whitney two-sided test. The relationship between markers and clinicopathological features was analyzed with the Pearson chi-square test. Survival analysis was undertaken using the log-rank test. The correlation between MLVD and claudin-1 expression was evaluated by Spearman correlation analysis. All the statistical analyses were two-sided tests and differences were considered to be statistically significant when $\mathrm{P}<0.05$.

\section{RESULTS}

\section{Immunohistochemistry of claudin-1 protein}

\section{Claudin-1 protein expressed in HSCC and adjacent tissues}

We subjected $97 \mathrm{HSCC}$ tissues and 90 adjacent tissues to immunohistochemistry staining and the results showed that claudin-1 was expressed both in the membrane and the cytoplasm, but was found mostly in the cytoplasm (around the nuclear membrane) (Figure 1A). Claudin-1 was diffused unevenly and located in different areas. Claudin-1 had a high expression of $73.2 \%$ $(71 / 97)$ in the cancer tissues, but only $30 \%$ (27/90) in the adjacent tissues. The difference was statistically significant.

\section{Correlation between claudin-1 expression in tumors and clinicopathological indices}

We conducted analyses using the Pearson chi-square test and the results showed that there was significant correlation (Table 2) between claudin-1 expression and tumor differentiation $(P=0.004)$ and lymph node metastasis $(P=0.026)$, while the other clinicopathological indices were not related to claudin-1 expression. 
Table 2. Relationship between claudin-1 expression and clinicopathological features.

\begin{tabular}{|c|c|c|c|c|}
\hline \multirow[t]{2}{*}{ Characteristics } & \multirow[t]{2}{*}{ No. of cases (\%) } & \multicolumn{3}{|c|}{ Claudin-1 expression } \\
\hline & & Low expression (\%) & High expression (\%) & $P$ \\
\hline \multicolumn{5}{|l|}{ Age (years) } \\
\hline$<60$ & $54(55.7)$ & $17(17.5)$ & 37 (38.1) & \\
\hline$\geq 60$ & $43(44.3)$ & $9(9.3)$ & 34 (35.1) & 0.175 \\
\hline \multicolumn{5}{|l|}{ Gender } \\
\hline Female & $9(9.3)$ & $5(5.2)$ & $4(4.1)$ & \\
\hline Male & $88(90.7)$ & $21(21.6)$ & $67(69.1)$ & 0.055 \\
\hline \multicolumn{5}{|c|}{ Tobacco consumption } \\
\hline Little or none & $19(19.6)$ & $8(8.2)$ & $11(14.3)$ & \\
\hline Excess & $78(80.4)$ & $18(18.6)$ & $60(61.9)$ & 0.085 \\
\hline \multicolumn{5}{|c|}{ Alcohol consumption } \\
\hline Little or none & $35(36.1)$ & $13(13.4)$ & $22(22.7)$ & \\
\hline Excess & $62(63.9)$ & $13(13.4)$ & $49(50.5)$ & 0.069 \\
\hline \multicolumn{5}{|l|}{ Differentiation } \\
\hline Good & $25(25.8)$ & $13(13.4)$ & $12(12.4)$ & \\
\hline Moderate & $37(38.1)$ & $7(7.2)$ & $30(30.9)$ & \\
\hline Poor & $35(36.1)$ & $6(6.2)$ & 29 (29.9) & 0.004 \\
\hline \multicolumn{5}{|l|}{ TNM stage } \\
\hline $1+11$ & $18(18.6)$ & $3(3.1)$ & $15(15.5)$ & \\
\hline III+IV & $79(81.4)$ & $23(23.7)$ & $56(57.7)$ & 0.221 \\
\hline \multicolumn{5}{|c|}{ Lymph node metastases } \\
\hline No & $35(36.1)$ & $14(14.4)$ & $21(21.6)$ & \\
\hline Yes & $62(63.9)$ & $12(12.4)$ & $50(51.5)$ & 0.026 \\
\hline
\end{tabular}

\section{Survival analysis}

The 97 patients with HSCC in our experiment were followed-up for 3-5 years. We conducted survival analysis using the log-rank test and found that claudin-1 was related to the patients' survival rate $(P=0.003)$ (Figure 2$)$.

\section{Survival proportions}

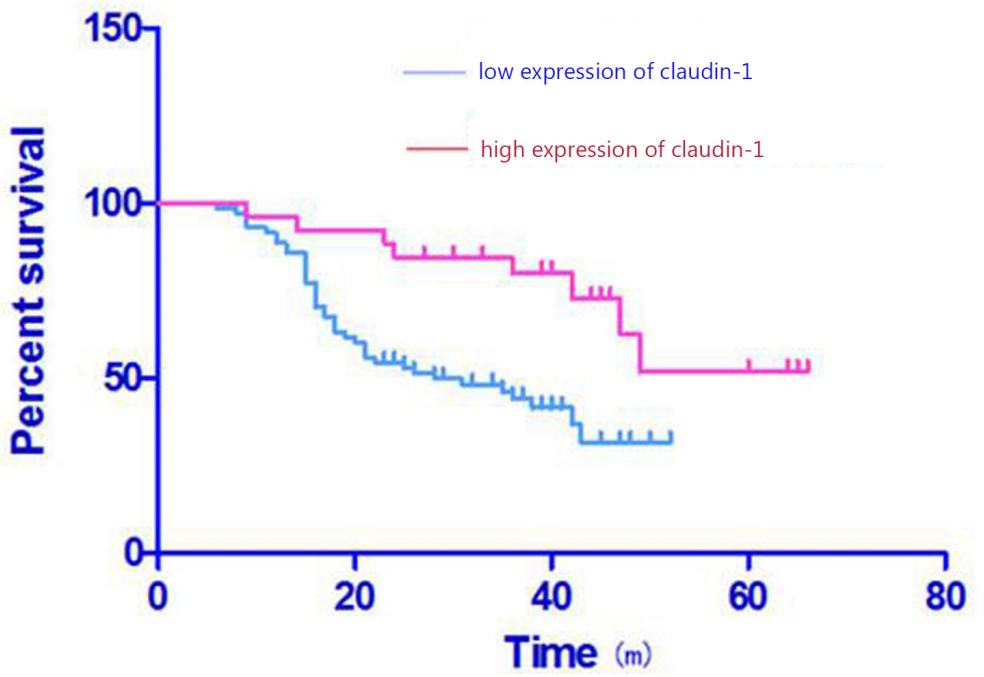

Figure 2. Relationship between claudin-1 and survival analysis of patients. 


\section{Immunohistochemistry of D2-40}

\section{Expression of D2-40 in lymphatic microvessels, and MLVD in HSCC and adjacent normal tissues}

D2-40 appeared as brown particles and was expressed in the cytoplasm of lymphatic microvessel endothelial cells. It was distributed in lymphatic microvessels with different morphologies in varying amounts around the central area, the peripheral region, and adjacent tissues of HSCC nests. Lymphatic microvessels were infrequent or absent around the cancer nests, and most took the form of collapsing strips (Figure 3A) with different morphologies and smaller lumens with thinner walls. However, around the peripheral region, most lymphatic microvessels appeared expansive and had various morphologies (Figure 3B); some had a tumor thrombus (Figure 3C). Around the adjacent normal tissues, the morphology of the lymphatic microvessels was more regular; most appeared expansive and were distributed in the submucosa (Figure 3D). In the 97 cases of HSCC specimens, the number of lymphatic microvessels around the adjacent tissue of cancer nests $(14.15 \pm 4.46)$ was significantly greater than in the central area $(3.35 \pm 2.32)(P<0.05)$. The number of lymphatic microvessels around the peripheral region of the cancer nests $(14.15 \pm 4.46)$ was also significantly greater than the number around the adjacent normal tissues $(4.52 \pm 2.65)(P<0.05)$. However, there was no significant difference between the central areas and the adjacent normal tissues $(P>0.05)$.
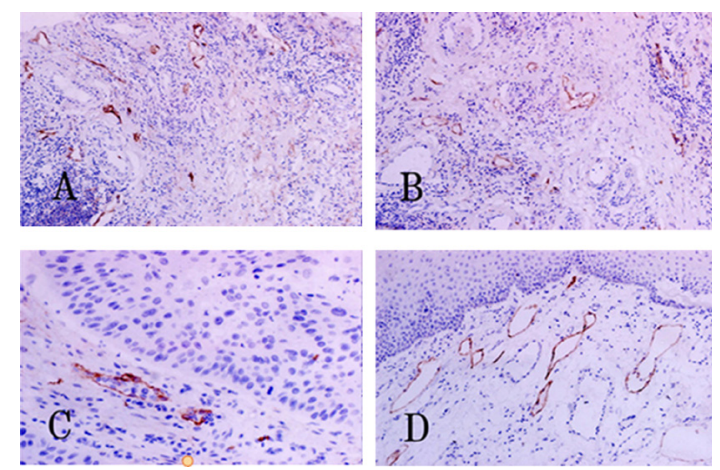

Figure 3. A. Number/distribution of lymphatic microvessels around the central area of the tumor nests (200X). B. expression of lymphatic microvessels around the peripheral region of tumor nests (200X). C. Tumor thrombus in the lymphatic microvessels around the peripheral region of tumor nests (400X). D. Expression of lymphatic microvessels around the adjacent normal tissues (200X).

\section{Relationship between MLVD and the clinical data}

The analysis of the correlation between the MLVD around the central area of the cancer nests and the indices [age, gender, tobacco consumption (little or none, or too much), alcohol consumption (little or none, or too much), differentiation, TNM stage, lymph node metastases] of the patients revealed no significant differences $(P>0.05)$ (Table 3).

The analysis of the correlation between the MLVD around the peripheral regions of the cancer nests and the indices of the patients revealed that there were no significant differences $(P$ $<0.05)$ except in the cases of differentiation, TNM stage, and lymph node metastases $(P=0.011$, $P=0.004$, and $P<0.001$, respectively). 
Table 3. Relationship between micro-lymphatic vessel density (MLVD) and clinical data in hypopharyngeal squamous cell carcinoma (HSCC).

\begin{tabular}{|c|c|c|c|c|c|}
\hline \multirow[t]{2}{*}{ Clinical data } & \multirow[t]{2}{*}{ No. of cases (\%) } & \multicolumn{4}{|c|}{ HSCC MLVD } \\
\hline & & Central area & $P$ & Peripheral region & $P$ \\
\hline \multicolumn{6}{|l|}{ Age (years) } \\
\hline$<60$ & $54(55.7)$ & $4.28 \pm 1.56$ & & $14.26 \pm 3.25$ & \\
\hline$\geq 60$ & $43(44.3)$ & $3.86 \pm 2.11$ & 0.856 & $13.98 \pm 2.56$ & 0.635 \\
\hline \multicolumn{6}{|l|}{ Gender } \\
\hline Female & $9(9.3)$ & $4.35 \pm 2.05$ & & $14.68 \pm 2.36$ & \\
\hline Male & $88(90.7)$ & $4.08 \pm 1.66$ & 0.639 & $14.22 \pm 3.06$ & 0.562 \\
\hline \multicolumn{6}{|c|}{ Tobacco consumption } \\
\hline Little or none & 19 (19.6) & $4.53 \pm 1.35$ & & $13.85 \pm 3.28$ & \\
\hline Excess & $78(80.4)$ & $4.11 \pm 2.05$ & 0.535 & $14.32 \pm 2.14$ & 0.322 \\
\hline \multicolumn{6}{|c|}{ Alcohol consumption } \\
\hline Little or none & $35(36.1)$ & $4.39 \pm 2.75$ & & $13.66 \pm 2.76$ & \\
\hline Excess & $62(63.9)$ & $4.57 \pm 1.78$ & 0.764 & $14.02 \pm 1.88$ & 0.207 \\
\hline \multicolumn{6}{|l|}{ Differentiation } \\
\hline Good & $25(25.8)$ & $3.95 \pm 2.06$ & & $12.58 \pm 2.45$ & \\
\hline Moderate & $37(38.1)$ & $4.24 \pm 1.44$ & & $14.60 \pm 3.22$ & \\
\hline Poor & $35(36.1)$ & $4.38 \pm 1.89$ & 0.636 & $16.65 \pm 3.56$ & 0.011 \\
\hline \multicolumn{6}{|l|}{ TNM stage } \\
\hline $1+I I$ & $18(18.6)$ & $3.75 \pm 2.83$ & & $13.33 \pm 2.74$ & \\
\hline III+IV & $79(81.4)$ & $4.32 \pm 2.23$ & 0.535 & $16.98 \pm 3.15$ & 0.004 \\
\hline \multicolumn{6}{|l|}{ LN metastases } \\
\hline No & 35 (36.1) & $4.15 \pm 2.36$ & & $12.62 \pm 2.25$ & \\
\hline Yes & $62(63.9)$ & $4.86 \pm 2.03$ & 0.606 & $17.32 \pm 3.69$ & $<0.001$ \\
\hline
\end{tabular}

\section{Mutual relationship between claudin-1 expression and MLVD}

The MLVD around the HSCC nests in the central areas was $4.68 \pm 1.42$ in cases of positive claudin-1 expression, which was not significantly different to the negative group (3.54 \pm 2.75) $(P>0.05)$ (Table 4).

The MLVD around the HSCC nests in the peripheral region was $16.58 \pm 3.36$ in cases of positive claudin-1 expression, which was significantly different to the negative group (12.16 \pm 2.54) $(P<0.05)$. Correlation analysis showed that there was a positive correlation between the claudin-1 expression around the HSCC nests in the peripheral region and MLVD $\left(r=0.54, X^{2}=\right.$ 9.6, $\mathrm{P}<0.05)($ Table 5).

Table 4. Relationship between claudin-1 expression and micro-lymphatic vessel density (MLVD) around hypopharyngeal squamous cell carcinoma (HSCC) nests in the central area.

\begin{tabular}{lcc}
\hline Claudin-1 expression (cases) & MLVD around the central area & $P$ \\
\hline Positive group (71) & $4.68 \pm 1.42$ \\
Negative group (26) & $3.54 \pm 2.75$ & $>0.05$ \\
\hline
\end{tabular}

Table 5. Relationship between claudin-1 expression and micro-lymphatic vessel density (MLVD) around hypopharyngeal squamous cell carcinoma (HSCC) nests in the peripheral region.

\begin{tabular}{lcc}
\hline Claudin-1 expression (cases) & MLVD around the peripheral region & $P$ \\
\hline Positive group (71) & $16.58 \pm 3.36$ \\
Negative group (26) & $12.16 \pm 2.54$ \\
\hline
\end{tabular}




\section{DISCUSSION}

The mechanism of claudin expression in tumor tissue has not been studied sufficiently. The abnormal expression of claudin leads to abnormal signal transduction between cells and promotes tumor generation and metastasis (Krause et al., 2008). The abnormal expression of claudin-1 destroys the osmotic barrier function of epithelial cells, removes the polarity of cells, and reduces the adhesion force between cells, which leads to tumor generation and development (Fritzsche et al., 2008). Furthermore, studies have shown that high expression of claudin-1 inhibits the expression of E-cadherin, which upregulates the E-cadherin/Tcf signal pathway. The change in the level of claudin-1 expression on the membrane, the decrease in E-cadherin expression, and the upregulation of the E-cadherin/Tcf signal pathway all promote tumor generation and development (Dhawan et al., 2005). High expression of claudin-1 has been detected in a variety of tumors (Soini and Talvensaari-Mattila, 2006; Fritzsche et al., 2008; Huo et al., 2009); in studies on oral squamous cell carcinoma, Dos Reis et al. (2008) found that high expression of claudin-1 was related to vessel invasion and peripheral nerve invasion in tumor tissues. In our study, claudin-1 expression in HSCC was significantly greater than in adjacent tissues, and there was a correlation between claudin-1 expression and both tumor differentiation $(P=0.004)$ and lymph node metastases $(P=$ $0.026)$, but there was no correlation with the other clinicopathological indices. The Kaplan-Meier analysis showed that the survival rate of patients with high claudin-1 expression was significantly lower than that of patients with low claudin-1 expression $(P=0.012)$. The reason for this difference in survival rates is thought to arise because high claudin-1 expression improves cell metastatic activity, with the activation of matrix metalloproteinase-2 (MMP-2), laminin-5 gamma-2 lysates, and epidermal growth factor (EGF) receptor (Oku et al., 2006). However, recent studies seem to show that the upregulation of MMP-2 expression improves tumor invasion capability, which leads to poor prognosis (de la Peña et al., 2010). These studies showed that claudin-1 might play a role in HSCC generation and development, and in lymph node metastasis, and may be used for HSCC diagnosis, prognostic evaluation, and to choose the appropriate treatment protocol. Patients with high claudin-1 expression levels require a higher level of intervention after operation.

D2-40, a salivary mucin comprising 166 amino acids, was discovered by Marks et al. (1999) in studies on germ cell tumors and seminoma of the fetal testis. As a monoclonal antibody, D2-40 can recognize and combine specifically to M2A antigen, expressed in the fetal testis and on the surface of testicular germ cell tumors. By immunohistochemistry double staining of D2-40 with vascular endothelial markers (CD34, CD31), Afonso et al. (2009) found that there was no cross reaction during combined staining of D2-40 and vascular endothelial markers. Hence, they considered using D2-40 to label lymphatic vessels possessing high specificity. Kahn et al. (2002) found that the D2-40 antibody could express specifically in lymphatic endothelial cells; although there was hemangioma, angiolipoma, vascular malformation, etc., there was no significant expression in angiopathy or around vessels; hence, the group considered that D2-40 was a specific marker of lymphatic endothelial cells and could be used to distinguish specifically whether a tumor was lymphogenic or angiogenic cerebral. Further investigation of the ultrastructure by electron microscopy revealed that D2-40 was expressed mainly on the cavity surface of lymphatic microvessel endothelial cells but not in lymphatic vessel endothelial cells or blood vessel endothelial cells (Braun et al., 2008), which showed that the specificity and sensitivity of D2-40 were higher than those of other lymphatic vessel markers (Yonemura et al., 2006).

In our studies, we found that in HSCC tissue, lymphatic microvessels labeled by D240 mainly aggregated around the peripheral regions of tumor nests and peripheral interstitial 
regions, while in vascular endothelial cells, there was no significant staining or cross reaction around the tissue. Labeling of MLVD showed significant specificity and better clinical effect. Most of the lymphatic microvessels labeled with D2-40 around the peripheral regions of HSCC nests appeared to have expansive lumens, with irregular morphology, without erythrocytes, and some of the lymphatic vessels had a tumor thrombus. However, the lymphatic microvessels of the HSCC nests were rare or completely absent. Most of the lumens were blocked and the morphology was atretic striation or flat tubular. The lymphatic microvessels in normal tissues adjacent to the carcinoma were more regular, and most of them had expansive lumens and were distributing in the submucosa. These results showed that D2-40 was specific to lymphatic endothelial cells in HSCC, and was a good marker for lymphatic microvessels.

Most of the lymphatic microvessels in the central areas and peripheral regions of the HSCC nests were irregular, with smaller lumens. The tube wall comprised two or three layers of endothelial cells, which were sometimes incomplete. The morphology of lymphatic microvessels in the central areas and peripheral regions of the HSCC nests were significantly different from those in carcinoma and adjacent normal tissues. Moreover, considering that they were immature lymphatic vessels, parts of them had a tumor thrombus, which meant that when HSCC occurred, new lymphatic microvessels were being generated, belonging to the neonatal lymphatic microvessels but not the existing one.

Beasley et al. (2002) thought that in head and neck squamous cell carcinoma, MLVD was high in tumors and was related to lymph node metastasis. They considered that the new lymphatic microvessels were functional and the tumor metastasized to lymph nodes through these lymphatic vessels. In studies of breast cancer, Skobe et al. (2001) found that the metastasis of new lymphatic vessels in tumors was related to regional lymph node metastasis and lung metastasis. The increase of lymphatic vessels in the tumor encouraged the tumor cells to leave their primary location and move into the lymphatic system. In our studies, we found that in HSCC, the MLVD of cancer nests in the peripheral region was significantly higher than in the central area (14.15 \pm 4.46 vs $3.35 \pm 2.32$ ). The MLVD of cancer nests in the peripheral region was related to lymph node metastasis; however, that was not the case in the central area. The explanation for this might be that with the over-generation and proliferation of tumor cells, the internal clearance gap pressure of the tumor tissues increased, leading to the lymphatic vessels in the central area of the carcinoma nests presenting high pressure status, and receiving the extrusion, invasion, and breaking of tumor cells, which led to compression, collapse, atrophy, and loss of function in the lymphatic vessels (Jain and Fenton, 2002). However, the pressure of tissue space around the carcinoma nests in the peripheral region was lower, with lower interstitial hydrostatic pressure, which was not sufficient to block the lymphatic microvessels. This caused most of the new lymphatic vessels to appear expansive, initiate lymphatic system hyperplasia, and turn into functional lymphatic vessels. All the studies provided advantageous conditions for tumor cell peripheral metastasis (Kandemir et al., 2012), provided tumor cells with nutrition by diffusion, and a direct metastatic channel. Tumor cells were able to arrive at the lymph nodes through these lymphatic vessels, thereby promoting lymph node metastasis of the tumor. In the studies by Padera et al. (2002), the lymphatic vessels in the tumor were broken by the tumor tissue, with only some endothelial cells remaining. Thus, the lymphatic vessels lost function and the tumor metastasized by invading functional lymphatic vessels adjacent to the tumor. This was consistent with our studies. Nitti et al. (2003) also considered that the lymphatic vessels in the tumor were non-functional and unrelated to the tumor metastasis, while the lymphatic microvessels adjacent to the tumor were functional, and could facilitate tumor metastasis. Therefore, we think that in HSCC the lymphatic vessels around the peripheral region of 
the carcinoma nests play an important role in metastasis in local lymph nodes, where the functional vessels lead to tumor metastasis. Detecting MLVD around carcinoma nests in the peripheral region could be regarded as an important index for predicting lymph node metastasis. Werner et al. (1990) thought that the blocking and decreasing of lymphatic microvessels in tumors could lead to the opening of a large number of lymphatic microvessels in the interstitial region around tumors. In HSCC, whether the lymphatic vessels around carcinoma nests in the peripheral region are related to the blocking of the lymphatic vessels around the carcinoma nests requires further research.

Yoon et al. (2010) found that the overexpression of claudin-1 enhanced the expression and activity of MMP-2. However, recent studies have revealed that MMP-2 can effectively promote the release and upregulation of vascular endothelial growth factor C (VEGF-C) and VEGF-D (Park et al., 2003). Acknowledged as the primary regulatory factors of lymph vessel generation, VEGF-C and VEGF-D exert biological effects by combining with the VEGF receptors VEGFR-2 and VEGFR-3. VEGF-C and VEFG-D regulate the generation of lymphatic vessels by combining with VEGFR-3. Shida et al. (2006) found that at low concentrations, VEGF-C and VEGF-D combined preferentially with VEGFR-3, which led specifically to hyperplasia of lymphatic vessels and generation of new lymphatic vessels by activating the MEK/P13-kinase/Akt pathway. Many studies have shown that VEGF-C and VEGF-D are closely related to the distant lymph node metastasis of many epitheliumderived tumors, and their associated prognosis (Skobe et al., 2001). Therefore, we consider that MMP-2 not only promotes the release and metastasis of lymphatic vessel endothelial cells by degrading the extracellular matrix, but also induces VEGF-C/D to combine with the receptor VEGFR-3 more effectively by enhancing the release and upregulation of VEGF-C/D, which induces the tyrosine phosphorylation of VEGF-C/D. This further induces the PI3-k signal transduction pathway, encouraging the activation of the ERK1/2 (p42/p44 MAPK) and Akt signal pathways and specifically acting on lymphatic vessel endothelial cells, thus initiating a cascade reaction, which leads to the differentiation, proliferation, and metastasis of lymphatic vessel endothelial cells. Finally, all of these initiate the proliferation of existing lymphatic vessels and the generation of new lymphatic vessels, providing advantageous conditions for tumor lymphatic metastasis.

Recent studies have shown that lymphatic microvessel generation induced by tumors is an important part of tumor lymphatic metastasis (Kumada et al., 2004). The new lymphatic microvessels, with a simple structure, are composed of a monolayer of endothelial cells, without a basal layer and fewer TJs between cells. All of these factors promote the migration of tumor cells into lymphatic vessels. Moreover, the generation of lymphatic microvessels leads to an increase in MLVD and a decrease in the adhesion force between cells. This induces the tumor cells to detach from their primary location, close, invade the lymphatic microvessels, metastasize to the regional lymph node through the lymphatic vessel, and finally form lymph node metastases (Kim and Dumont, 2003).

Claudin-1 is a TJ protein, whereas E-cadherin is a cell adhesion protein that is involved in the primary adhesion system, the TJ and adherent junction, as the molecular basis for adhesion between homotype cells. Both proteins are expressed on the surface of most epithelial cells. They maintain the polarity, integrity, stability, morphology, and structure of epithelial cells by mediating cell-extracellular matrix and cell-cell adhesion and specific signal transduction. They thereby maintain the normal cell phenotype, influence cell migration, and play an important role in inhibiting tumor generation and development (Yang et al., 2009). However, high expression of claudin-1 can inhibit the expression of E-cadherin, which upregulates the E-cadherin/Tcf signal pathway; together, these three factors affect tumor generation and development (Dhawan et al., 2005). In our studies on HSCC, the MLVD was significantly higher in the group with positive expression of claudin-1 
than in the group with negative expression. We consider that the overexpression of claudin-1 leads to the release and upregulation of VEGF-C and VEGF-D by enhancing MMP-2 expression, and through a particular pathway, it can specifically induce the hyperplasia of lymphatic vessels and the generation of new lymphatic vessels. At the same time, the overexpression of claudin-1 can inhibit the expression of E-cadherin, which leads to a reduction in cell-cell adhesion. As mentioned above, this ultimately leads to the formation of lymph node metastases.

In conclusion, we found that claudin-1 was overexpressed in HSCC and was related to the differentiation of tumor cells and lymph node metastasis. At the same time, the MLVD in the group with positive claudin-1 expression was higher than in the negative group. We consider that the overexpression of claudin-1 can induce the generation of tumor lymphatic vessels, thus promoting lymph node metastasis. Moreover, claudin-1 is related to patient survival rate; it may be useful as a postoperative monitoring index for $\mathrm{HSCC}$ and might be a new target for treating the disease.

\section{Conflicts of interest}

The authors declare no conflict of interest.

\section{REFERENCES}

Afonso J, Santos LL, Amaro T, Lobo F, et al. (2009). The aggressiveness of urothelial carcinoma depends to a large extent on lymphovascular invasion - the prognostic contribution of related molecular markers. Histopathology 55: 514-524.

Beasley NJ, Prevo R, Banerji S, Leek RD, et al. (2002). Intratumoral lymphangiogenesis and lymph node metastasis in head and neck cancer. Cancer Res. 62: 1315-1320.

Braun M, Flucke U, Debald M, Walgenbach-Bruenagel G, et al. (2008). Detection of lymphovascular invasion in early breast cancer by D2-40 (podoplanin): a clinically useful predictor for axillary lymph node metastases. Breast Cancer Res. Treat. 112: 503-511.

Chao YC, Pan SH, Yang SC, Yu SL, et al. (2009). Claudin-1 is a metastasis suppressor and correlates with clinical outcome in lung adenocarcinoma. Am. J. Respir. Crit. Care Med. 179: 123-133.

de la Peña S, Sampieri CL and León-Córdoba K (2010). Matrix metalloproteases as molecular markers in gastric cancer. Med. Clin. 134: 123-126.

Dhawan P, Singh AB, Deane NG, No Y, et al. (2005). Claudin-1 regulates cellular transformation and metastatic behavior in colon cancer. J. Clin. Invest. 115: 1765-1776.

Dos Reis PP, Bharadwaj RR, Machado J, Macmillan C, et al. (2008). Claudin 1 overexpression increases invasion and is associated with aggressive histological features in oral squamous cell carcinoma. Cancer 113: 3169-3180.

Folkman J (1995). Angiogenesis in cancer, vascular, rheumatoid and other disease. Nat. Med. 1: 27-31.

Fritzsche FR, Oelrich B, Johannsen M, Kristiansen I, et al. (2008). Claudin-1 protein expression is a prognostic marker of patient survival in renal cell carcinomas. Clin. Cancer Res. 14: 7035-7042.

Harhaj NS and Antonetti DA (2004). Regulation of tight junctions and loss of barrier function in pathophysiology. Int. J. Biochem. Cell Biol. 36: 1206-1237.

Heiskala M, Peterson PA and Yang Y (2001). The roles of claudin superfamily proteins in paracellular transport. Traffic 2: 93-98.

Huo Q, Kinugasa T, Wang L, Huang J, et al. (2009). Claudin-1 protein is a major factor involved in the tumorigenesis of colorectal cancer. Anticancer Res. 29: 851-857.

Ishida M, Kushima R and Okabe H (2009). Claudin expression in rectal well-differentiated endocrine neoplasms (carcinoid tumors). Oncol. Rep. 21: 113-117.

Jain RK and Fenton BT (2002). Intratumoral lymphatic vessels: a case of mistaken identity or malfunction? J. Natl. Cancer Inst. 94: 417-421.

Johnson JT, Bacon GW, Myers EN and Wagner RL (1994). Medial vs lateral wall pyriform sinus carcinoma: implications for management of regional lymphatics. Head Neck 16: 401-405.

Kahn HJ, Bailey D and Marks A (2002). Monoclonal antibody D2-40, a new marker of lymphatic endothelium, reacts with Kaposi's sarcoma and a subset of angiosarcomas. Mod. Pathol. 15: 434-440.

Kandemir NO, Barut F, Bektas S and Ozdamar SO (2012). Can lymphatic vascular density be used in determining metastatic 
spreading potential of tumor in invasive ductal carcinomas? Pathol. Oncol. Res. 18: 253-262.

Kim H and Dumont DJ (2003). Molecular mechanisms in lymphangiogenesis: model systems and implications in human disease. Clin. Genet. 64: 282-292.

Krause G, Winkler L, Mueller SL, Haseloff RF, et al. (2008). Structure and function of claudins. Biochim. Biophys. Acta. 1778: 631-645.

Kumada T, Tsuneyama K, Hatta H, Ishizawa S, et al. (2004). Improved 1-h rapid immunostaining method using intermittent microwave irradiation: practicability based on 5 years application in Toyama Medical and Pharmaceutical University Hospital. Mod. Pathol. 17: 1141-1149.

Liang H, Giorgadzc R, Bcllucci K, Veerappan R, et al. (2005). D2-40 is highly expressed in primary skin adnexal carcinomas but negative in adenocarcinoma metastases to skin. Mod. Pathol. 18: 86A.

Marks A, Sutherland DR, Bailey D, Iglesias J, et al. (1999). Characterization and distribution of an oncofetal antigen (M2A antigen) expressed on testicular germ cell tumours. Br. J. Cancer 80: 569-578.

Mazzone A, Califano L, Agozzino L, Barillari U, et al. (1990). Anatomopathologic and clinical correlations of squamous carcinoma of the larynx and the hypopharynx. Acta Otorhinolaryngol. Ital. 10: 111-119.

Nitti D, Marchet A, Olivieri M, Ambrosi A, et al. (2003). Ratio between metastatic and examined lymph nodes is an independent prognostic factor after D2 resection for gastric cancer: analysis of a large European monoinstitutional experience. Ann. Surg. Oncol. 10: 1077-1085.

Oku N, Sasabe E, Ueta E, Yamamoto T, et al. (2006). Tight junction protein claudin-1 enhances the invasive activity of oral squamous cell carcinoma cells by promoting cleavage of laminin- 5 gamma2 chain via matrix metalloproteinase (MMP)-2 and membrane-type MMP-1. Cancer Res. 66: 5251-5257.

Padera TP, Kadambi A, di Tomaso E, Carreira CM, et al. (2002). Lymphatic metastasis in the absence of functional intratumor lymphatics. Science 296: 1883-1886.

Park DY, Sol MY, Suh KS, Shin EC, et al. (2003). Expressions of transforming growth factor (TGF)-beta1 and TGF-beta type II receptor and their relationship with apoptosis during chemical hepatocarcinogenesis in rats. Hepatol. Res. 27: 205-213.

Pingree TF, Davis RK, Reichman O and Derrick L (1987). Treatment of hypopharyngeal carcinoma: a 10-year review of 1,362 cases. Laryngoscope 97: 901-904.

Shida A, Fujioka S, Kobayashi K, Ishibashi Y, et al. (2006). Expression of vascular endothelial growth factor (VEGF)-C and -D in gastric carcinoma. Int. J. Clin. Onco1. 11: 38-43.

Skobe M, Hawighorst T, Jackson DG, Prevo R, et al. (2001). Induction of tumor lymphangiogenesis by VEGF-C promotes breast cancer metastasis. Nat. Med. 7: 192-198.

Soini $Y$ and Talvensaari-Mattila A (2006). Expression of claudins 1, 4, 5, and 7 in ovarian tumors of diverse types. Int. J. Gynecol. Pathol. 25: 330-335.

Tökés AM, Kulka J, Paku S, Szik A, et al. (2005). Claudin-1, -3 and -4 proteins and mRNA expression in benign and malignant breast lesions: a research study. Breast Cancer Res. 7: R296-R305.

Wang KH, Brose K, Arnott D, Kidd T, et al. (1999). Biochemical purification of a mammalian slit protein as a positive regulator of sensory axon elongation and branching. Cell 96: 771-784.

Werner JA, Schünke M, Rudert H and Tillmann B (1990). Description and clinical importance of the lymphatics of the vocal fold. Otolaryngol. Head Neck Surg. 102: 13-19.

Yang ZQ, Huang YC, Zou TN, Li M, et al. (2009). E-cadherin, claudin-1 expression and clinical significance in breast cancer. The Pract. J. Cancer 24: 639-640.

Yonemura Y, Endou Y, Tabachi K, Kawamura T, et al. (2006). Evaluation of lymphatic invasion in primary gastric cancer by a new monoclonal antibody, D2-40. Hum. Pathol. 37: 1193-1199.

Yoon CH, Kim MJ, Park MJ, Park IC, et al. (2010). Claudin-1 acts through c-Abl-protein kinase Cdelta (PKCdelta) signaling and has a causal role in the acquisition of invasive capacity in human liver cells. J. Biol. Chem. 285: 226-233. 